\title{
REAL TIME TURBULENCE ESTIMATION USING DOPPLER LIDAR MEASUREMENTS Lucie Rottner ${ }^{1 *}$, Christophe Baehr ${ }^{1}$ \\ ${ }^{1}$ Météo-France, CNRM-GAME, Toulouse, France. *lucie.rottner@meteo.fr
}

\begin{abstract}
A preliminary work on a new way to estimate atmospheric turbulence using high-frequency Doppler lidar measurements is presented. The turbulence estimations are based on wind reconstruction using 3D Doppler lidar observations and a particle filter. The suggested reconstruction algorithm links the lidar observations to numerical particles to obtain turbulence estimations every time new observations are available. The high frequency of the estimations is a new point which is detailed and discussed. Moreover, the presented algorithm ables to reconstruct the wind in three dimensions in the observed volume. We have thus locally access to the spatial variability of the turbulent atmosphere. The suggested algorithm is applied to a set of real observations. The obtained results are very encouraging : they show significant improvements on turbulent parameter estimations.
\end{abstract}

\section{INTRODUCTION}

Turbulent phenomena in the Atmospheric Boundary Layer (ABL) are characterized by small spatial and temporal scales which make them difficult to observe and to model. As Doppler lidar provide fine and high-frequency observations, they are instruments of predilection to study wind and turbulence in the ABL. With the development of wind farms and the constant growth of traffic on airports, the need for turbulence estimations is growing. Lot of different parameters have been studied to estimate turbulence. In this paper, the novelty is not about the turbulent parameters themselves. Instead of working on these parameters we suggest a new way to reconstruct the wind from the observations. The reconstruction algorithm is a nonlinear Bayesian estimation method based on wind observations. Using the algorithm, the 3D wind is reconstructed and atmospheric turbulent parameters are estimated in a conic volume. The estimation method is based on a particle filter. Using this filter, the wind observations are associated to particle systems driven by a local Lagrangian turbulence model. The particles have both fluid and stochastic properties. Here is the innovation of our method : spatial averages and covariances may be deduced from the particles themselves. Thus wind gusts and turbulent structures may be detected in real time. The theoretical framework for turbulence estimations have been defined by Baehr [1] and then applied to vertical lidar [2]. A lidar WindCube is used to get 3D estimations. The WindCube geometry is described. This description leads to one assumption : the atmosphere is assumed to be locally homogeneous. Next the particle filter is introduced. Then the turbulent kinetic energy computation is discussed. Finally an application of the reconstruction algorithm to real WindCube observations is presented. The results show good wind reconstructions. For the turbulent parameter estimations, the main new point is the frequency of the estimations : turbulent parameters are estimated at the observation frequency.

\section{WINDCUBE GEOMETRY}

Wind reconstruction and turbulent parameter estimation are based on high-frequency and highquality wind observations. To get such observations, a lidar WindCube provided by LEOSPHERE is used. It is a heterodyne pulsed Doppler lidar which has a specific geometry. It has five different lines of sight. There is one vertical line surrounded by four oblique lines with an oblique angle of $28^{\circ}$ (see figure 1). After each emission of the lidar beam, the lidar gets back 10 observations with a spacing of 20 meters. The lidar performs measures one line after an other. A full revolution takes 4 seconds. The lidar has a maximum range of 220 meters covering the low ABL. Thanks to these characteristics, the lidar instrument allows to 


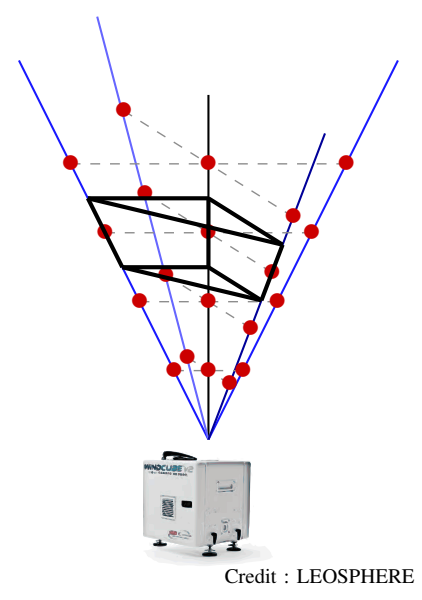

FiguRE 1: Lidar WindCube : 1 vertical line of sight and 4 oblique lines. The observed volume is split in boxes. One of them is drawn is black.

observe inertial turbulent scales in the ABL.

We may notice that as the lidar uses the Doppler effect to measure wind speed, it only observes the projection of the wind following the line of sight. So, to reconstruct the wind we have to use three observations following three different lines. We logically choose two consecutive observation points on a same vertical level and the vertical observation point related to this level. It makes appear an hypothesis : in order to reconstruct the turbulent atmosphere, we assume that the atmosphere is homogeneous between these three points and during a pattern repeat period. It is the assumption of locally homogenous medium. The observed volume is split in boxes. Each box contains three observations of radial wind (see fig. 1). Thus the atmosphere is considered homogenous in each box. This partition is usefull to compute turbulence estimations.

\section{PARTICLE FILTER}

When a set of data is collected, the usual question is to estimate the real state of a system using one or more observations at different time steps. It is the principle of data assimilation. The reconstruction of the turbulent atmosphere may be seen as an assimilation process : the wind observation is assimilated in the particle model. For each time step $k$, the state vector of the system $\xi_{k}$ is associated to the observation vector $y_{k}$, where the dimension of the vector $y_{k}$ is often smaller than the dimension

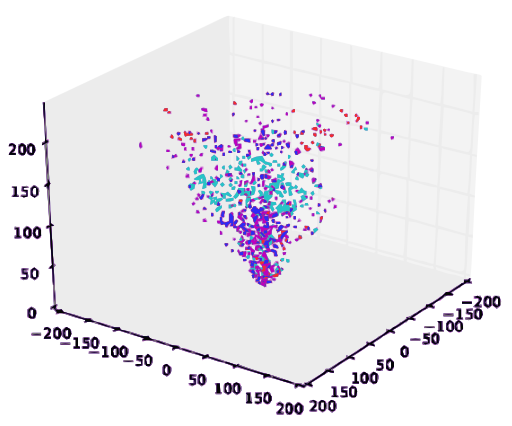

FIGURE 2: Illustration of a particle system filling a conic volume. The colored dots are the numerical particles.

of the state vector $\xi_{k}$. Then some coordinates of the vector $\xi_{k}$ are hidden for the observation $y_{k}$. Assuming that $\xi_{k}$ is a Markov process and the observational noise is a random variable, the classical problem consists in evaluating the probability density function (pdf) :

$$
p\left(\xi_{k} \mid y_{1: k}\right)=\frac{p\left(y_{k} \mid \xi_{k}\right) \cdot p\left(\xi_{k} \mid y_{1: k-1}\right)}{p\left(y_{k} \mid y_{1: k-1}\right)}
$$

with the following time recursion due to the Markov property

$p\left(\xi_{k} \mid y_{1: k-1}\right)=\int p\left(\xi_{k} \mid \xi_{k-1}\right) p\left(\xi_{k-1} \mid y_{1: k-1}\right) d \xi_{k-1}$.

For usual data assimilation, the evaluation of the expectation of $p\left(\xi_{k} \mid y_{1: k}\right)$ is based on variational methods and a meteorological model is used for the time prediction. Here the whole pdf is described using particle filters (PF) designed to study nonlinear processes. A PF uses a set of numerical particles to describe the probability density function (pdf) of interest. The observed volume is filled with the numerical particles. They follow the air flow and carry the local characteristics of the atmosphere. The PF is made of two sequential steps. First there is the evaluation of the pdf $p\left(\xi_{k} \mid y_{1: k}\right)$. The particle velocities are updated using the last observations : these updated particles are samples of the pdf $p\left(\xi_{k} \mid y_{1: k}\right)$. This resampling is called "importance sampling". Then there is a Markovian time prediction. Here, a physical model is used to predict the state at the next time step. The model is locally consistent with the Kolmogorov K41 laws and has been first introduced for turbulence estimation by [1]. Thus using the particle representation 
at the time step $k$, the pdf is approximated by :

$$
p\left(\xi \mid y_{1: k}\right) \sim p^{N}\left(\xi \mid y_{1: k}\right)=\frac{1}{N} \sum_{i=1}^{N} \delta\left(\xi-\xi_{k}^{i}\right)
$$

where $N$ is the number of particles and $\xi_{k}^{i}$ is the particle $i$ at the time step $k$.

\section{MEASURING TURBULENCE}

To estimate atmospheric turbulence, a usual parameter is the turbulent kinetic energy (TKE). The TKE is calculated using the variance of the wind velocity fluctuation. Let $\mathbf{V}=(u, v, w)$ be the 3D velocity vector. Following the Reynolds decomposition, $\mathbf{V}$ may be split in a mean component $\overline{\mathbf{V}}$ and a fluctuation $\mathbf{V}^{\prime}, \mathbf{V}=\overline{\mathbf{V}}+\mathbf{V}^{\prime}$. From this split, we can define the turbulent kinetic energy :

$$
\mathrm{TKE}=\frac{1}{2}\left(\overline{u^{\prime 2}}+\overline{{v^{\prime}}^{2}}+\overline{{w^{\prime}}^{2}}\right)
$$

Ideally the averaging operator should be determined by ensemble averaging. As the ensemble average is unreachable in usual pratical situations, time averaging is used instead. To use time averaging, two hypotheses are made : the turbulent flow is assumed ergodic and stationary. In this framework, time average and spatial average are equilvalent. Therefore, the TKE is obtained using the variance of the $3 \mathrm{D}$ wind speed $\mathbf{V}$ over a period $T$. At each observation point, the classical TKE is given in discrete time by :

$$
\mathrm{TKE}=\frac{1}{T} \sum_{k=1}^{T}\left(\mathbf{V}_{k}-<\mathbf{V}>_{T}\right)^{2}
$$

where $\langle.\rangle_{T}$ is the time average. To mesure the TKE using a lidar, the integration time $T$ has to be specified. Pichugina and O'Connor suggest methods to estimate the TKE and the eddy dissipation rate using a Doppler lidar [4] [3]. Following their works, to compute the TKE with a time average we choose $T=10 \mathrm{~min}$. This TKE is used as a reference in this paper. Here using the particle system, average and variance can be computed using the particle values. It leads that the suggested method overcomes the ergodicity and stationarity hypotheses. At any time, the particles represent the wind and its spatial variability. Thus the local wind speed variance is computed at each time step $k$. For $N$ particles close to the observation point, the TKE is given by :

$$
\operatorname{TKE}(k)=\frac{1}{N} \sum_{i=1}^{N}\left(\mathbf{V}_{k}^{i}-<\mathbf{V}_{k}>_{N}\right)^{2}
$$

where $<.>_{N}$ is the spatial average computed using the updated particles. At each time step $k$, the TKE is estimated in each box used to reconstruct the wind. We have thus access to the spatial and time variability of the turbulence.

\section{RESULTS}

To get relevant turbulent estimations, the first step is to assess the wind reconstruction. The aim is to reconstruct the 3D wind in the observed volume using the particle filter. The WindCube data have been provided by LEOSPHERE. The results are given in the classical frame. The observed wind is obtained by geometric construction using the three observations. We illustrate the results only in one box with the horizontal wind following the first coordinate $u$. The figure 3 shows the time series of the observed wind and the reconstructed wind over a $30 \mathrm{~min}$ period with a $4 \mathrm{~s}$ time step. The reconstructed serie can barely be separated from the observed one. The power spectrum densities (PSD) are presented on figure 4 . They confirm that the reconstructed wind follows the K41 Kolmogorov laws for the inertial sub-range turbulence. The K41 energy cascade is represented by a $-5 / 3$ slope drawn in green. The estimated wind is thus coherent with the observations. Its realistic shape is validated by the PSD which follows the energy cascade. The validation of the 3D wind estimations leads us to the estimations of turbulent parameters. The graph 5 illustrates the contribution of the particle method for turbulence estimation. For one box, it shows over a $2 \mathrm{~h}$ period with a 4 s time step the classical TKE calculated on 10 minutes and the TKE estimated at each time step with the particle method. The usual TKE is made of 10-minute long steps, whereas the particle system gives back a TKE made of irregular peaks. It seems that particles capture a spatial and time variability of the atmospheric turbulence which is clearly unreachable so far. However, even if the particle TKE shape is coherent with the intermittent nature of the turbulence, this estimation method has still to be validated. The natural approach to validate the particle TKE estimations is to average them on 10minute period and then to compare average particle TKE with classical TKE. In average, the particle 


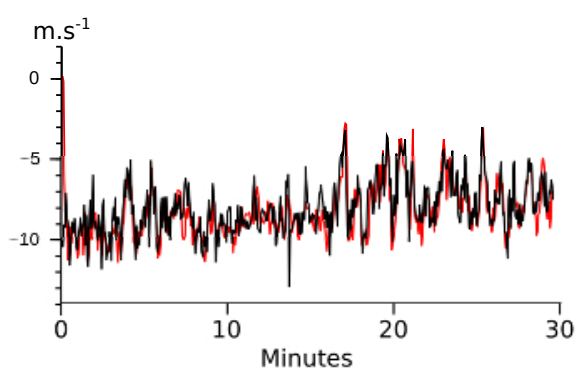

FIGURE 3: Time series of the horizontal reconstructed wind (in red) and the horizontal observed (by lidar) wind (in black), with a 4 s time step.

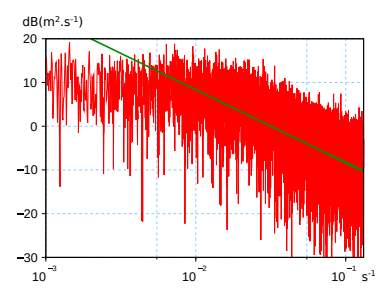

Figure 4: Power spectrum densities of the horizontal reconstructed wind. The Kolmogorov energy cascade is drawn in green.

TKE estimations are coherent with the classical estimations (not shown). However, an average validation is not enough to validate the peaky shape of the instantaneous particle TKE. As this variability is unreachable with classical methods, we have no way to provide further validations of instentaneous turbulence estimations.

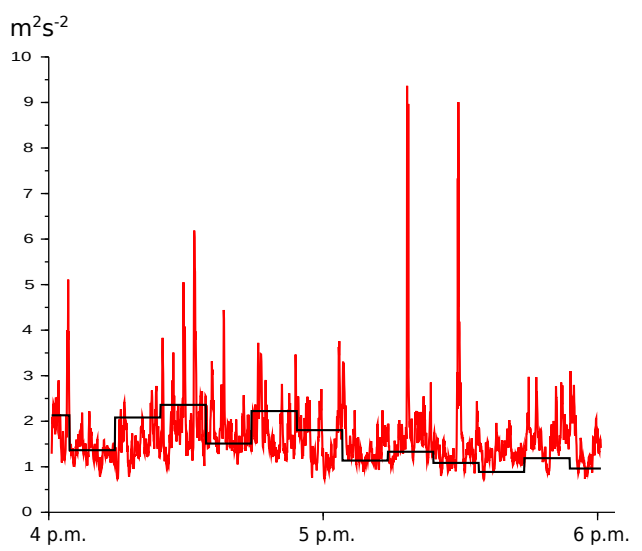

FIGURE 5: Usual TKE (black) versus particle TKE (red), for the 08/26/2010 from a test site in Greece.

\section{CONCLUSION}

A new way to estimate turbulent parameters has been introduced. Using our method the wind and the turbulent structure advection may be visualized in real time. The reconstruction algorithm have been applied on lidar WindCube measurements. The WindCube has been used because of its conic configuration. This configuration allows to reconstruct the wind in $3 \mathrm{D}$ and to estimate turbulent parameters in the observed volume. To validate the algorithm we have compared the estimated wind to the observed wind. The results show that the algorithm is able to reconstruct the 3D wind. We have then worked on TKE estimations. We have seen that the reconstruction algorithm brings completely new information on turbulence. Thanks to the association of highfrequency observations with particle methods, we have now access to local reconstruction of the turbulent atmosphere. Fine turbulence estimations are thus available. Experiences on real data show very good results. However the validation of the high-frequency turbulence estimations is still an open question as there is no way to assess the time variability of turbulent parameters so far.

\section{ACKNOWLEDGEMENT}

The author would like to thanks LEOSPHERE for providing the WindCube data.

\section{REFERENCES}

[1] C. Baehr. Nonlinear filtering for observations on a random vector field along a random path. application to atmospheric turbulent velocities. ESAIM : Mathematical Modelling and Numerical Analysis, 44(05) :921-945, 2010.

[2] C. Baehr, B. Claire, C. Fleur, D. Alain, and P. Bruno. Retrieval of the turbulent and backscattering properties using a nonlinear filtering technique applied to doppler lidar observation. In ISARS, 2012.

[3] E. J. O'Connor, A. J. Illingworth, I. M. Brooks, C. D. Westbrook, R. J. Hogan, F. Davies, and B. J. Brooks. A method for estimating the turbulent kinetic energy dissipation rate from a vertically pointing doppler lidar, and independent evaluation from balloon-borne in situ measurements. Journal of Atmospheric and Oceanic Technology, 27(10) :1652-1664, 2010.

[4] Y. L. Pichugina, S. C. Tucker, R. M. Banta, W. A. Brewer, N. D. Kelley, B. J. Jonkman, and R. K. Newsom. Horizontal-velocity and variance measurements in the stable boundary layer using doppler lidar : Sensitivity to averaging procedures. Journal of Atmospheric and Oceanic Technology, 25(8) :1307-1327, 2008. 